\title{
Increased expression levels of ppGalNAc-T13 in lung cancers: Significance in the prognostic diagnosis
}

\author{
KENICHI NOGIMORI ${ }^{1,3}$, TOMOKO HORI $^{1}$, KOJI KAWAGUCHI $^{4}$, TAKAYUKI FUKUI $^{4}$, \\ SHINJI MII ${ }^{5}$, HIROSHI NAKADA ${ }^{6}$, YASUYUKI MATSUMOTO ${ }^{1}$, YOSHIO YAMAUCHI ${ }^{1}$, \\ MASAHIDE TAKAHASHI ${ }^{5}$, KEIKO FURUKAWA ${ }^{2}$, OKAJIMA TETSUYA ${ }^{1}$, KOHEI YOKOI $^{4}$, \\ YOSHINORI HASEGAWA ${ }^{3}$ and KOICHI FURUKAWA ${ }^{1,2}$
}

\begin{abstract}
${ }^{1}$ Department of Biochemistry II, Nagoya University Graduate School of Medicine, Nagoya; ${ }^{2}$ Faculty of Life and Health Sciences, Chubu University, Kasugai; ${ }^{3}$ Department of Respiratory Medicine, Nagoya University Graduate School of Medicine, Nagoya; ${ }^{4}$ Department of Thoracic Surgery, Nagoya University Graduate School of Medicine, Nagoya; ${ }^{5}$ Department of Pathology, Nagoya University Graduate School of Medicine, Nagoya;

${ }^{6}$ Department of Molecular Bioscience, Faculty of Life Sciences, Kyoto Sangyo University, Kyoto, Japan
\end{abstract}

Received April 1, 2016; Accepted May 25, 2016

DOI: $10.3892 /$ ijo.2016.3638

\begin{abstract}
GalNAc-T13 is upregulated along with reduced expression of GM1 in high metastatic sublines of the murine Lewis lung cancercell line, but little is known about the implication of ppGalNAc-T13 expression in human cancers. Since lung cancer cell lines showed high expression levels of ppGalNAcT13, we analyzed ppGalNAc-T13 expression in surgical lung cancer specimens to examine whether ppGalNAc-T13 can be used as a prognostic marker or a therapeutic target. We analyzed mRNA expression levels of GALNT13 and its variant exon usages in surgical specimens by real-time RT-PCR, and the results were evaluated by correlating with clinical data. Ninety-one surgical specimens were analyzed. Consequently, recurrence-free survival was significantly shorter $(\mathrm{P}=0.045)$ in high expression group of GALNT13 mRNA. In the analysis of tumor specific exon usage in GALNT13 RNA sequence, one variant exon was significantly associated with worse prognosis. By contrast, in another variant exon, positive variant expression group showed better prognosis than negative group. We also tried to detect GALNT13 mRNA in 63 serum samples from patients with lung cancers to examine
\end{abstract}

Correspondence to: Professor Koichi Furukawa, Faculty of Life and Health Sciences, Chubu University, Kasugai, 1200 Matsumoto, Kasugai, Aichi 487-8501, Japan

E-mail: koichi@isc.chubu.ac.jp

Abbreviations: PCR, polymerase chain reaction; RT-PCR, reverse transcription polymerase chain reaction; qPCR, quantitative polymerase chain reaction; PBS, phosphate-buffered saline; BSA, bovine serum albumin; FBS, fetal bovine serum; T13, ppGalNAc-T13

Key words: ppGalNAc-T13, lung cancer, tumor marker, prognosis, metastasis whether GALNT13 mRNA can be measured in body fluids, detecting significant levels in 4 samples. Finally, expression of GM1, ppGalNAc-T13 and trimeric Tn antigen was examined by immunohistochemistry in order to evaluate them as a prognostic factor. It was demonstrated that ppGalNAc-T13 and trimeric $\mathrm{Tn}$ antigen had a relationship with worse prognosis in 35 investigated lung cancer patients. In conclusion, our results suggest that ppGalNAc-T13 might be a useful prognostic factor of lung cancers.

\section{Introduction}

Lung cancer is the most frequent malignancy and the prognosis is still poor (1). One of the reasons for worse prognosis is that tumor metastasis is often seen at the diagnosis. In addition, despite development of some molecularly-targeted drugs that target for EGFR-mutation or ALK-rearrangement, there are certain patients who cannot get these benefits $(2,3)$. Therefore, there is a strong need to define new therapeutic targets and biomarkers.

As mentioned above, metastasis is a major cause of death in lung cancer patients. In metastasis process, glycolipids and glycoproteins play important roles and are involved in cancer properties (4-7). Gangliosides, a kind of glycosphingolipid, are widely expressed in many tissues (8), but some gangliosides are very limitedly expressed in normal tissues, and their expression is enhanced in tumors. GD2 is highly expressed in small cell lung cancers, and is strongly associated with the malignant potential $(9,10)$. Furthermore, anti-GD2 monoclonal antibodies induce apoptosis of small cell lung cancer cells (11). NeuGcGM3 is overexpressed in non-small cell lung cancer and an anti-idiotype vaccine targeting the NeuGcGM3 is effective in the treatment of patients with advanced non-small cell lung cancer (12). As for carbohydrates on glycoproteins, O-glycans also play important roles in the malignancy and survival of cancer cells (6). O-glycans are often highly sialylated in cancer cells $(13,14)$. Among them, Tn antigen, which is 
Table I. Sequences of primers used in nested and real-time RT-PCR.

\begin{tabular}{llc}
\hline Gene & Forward primer & Reverse primer \\
\hline GALNT13 wild & 5'-TGGCCAGTGATTTGATTGCC-3' & 5'-AACGTGCATTCACAGTGTGC-3' \\
E5 & 5'-TGACTTTCCCTGCTTTCGTG-3' & 5'-ATGACCTGCCCTTTTGAAGC-3' \\
E13' & 5'-GTTACTTGCTCCCATGTTGGTC-3' & 5'-CAGGTCCATTGAGTCTGATTTC-3' \\
E14 & 5'-TGGGAATATGATGCTGAGTCTTG-3' & 5'-TTCATGTGCCCAAGGTCATG-3' \\
GALNT13 nested & & \\
Outer & 5'-GCATTGAGGGCTGTTATTTCAAG-3' & 5'-AGAGTAGATAGTGTGGGGAACG-3' \\
Inner & 5'-AAGAAGGGCCAGGAGAAATGG-3' & 5'-AGAGTGCTCCAAGCTTCATTATG-3' \\
\hline
\end{tabular}

formed by polypeptide $\mathrm{N}$-acetylgalactosaminyltransferases (ppGalNAc-T), and sialyl Tn antigen have been considered as tumor-associated antigens, and the expression levels of them are increased in many types of human cancers $(15,16)$.

In the analysis of mechanisms for cancer metastasis, we previously demonstrated that reduced levels of ganglioside GM1 resulted in the increased invasion and metastatic potentials (17). We also found that ppGalNAc-T13 (T13), which is a family member of ppGalNAc transferases, was upregulated as a result of reduced GM1, leading to enhanced metastasis by formation of trimeric Tn antigen on Syndecan 1 in mouse Lewis lung cancer (18-20). In human, it has been reported that GALNT13 mRNA is a strong predictor of poor clinical outcome in neuroblastoma patients (21). However, little is known about the relationship of the T13 expression and the phenotypes and prognosis in lung cancer patients. If the correlation between GALNT13 gene expression levels in lung cancer tissues and patient prognosis is indicated, it can be expected as a new prognostic marker and a therapeutic target. In addition, if it is detected in blood, it can be a useful biomarker for early detection or minimal metastatic diseases.

In the present study, T13 expression levels in surgical lung cancer specimens were analyzed to examine whether T13 and its product can be used as a tumor prognostic marker.

\section{Materials and methods}

Sample collection. Ninety-one patients received pulmonary resection at Nagoya University Hospital (2008, January2009, March). Before operation, each patient's serum sample was collected. The lung cancer tissues and sera were stored at $-80^{\circ} \mathrm{C}$. The treatment policy was decided according to the standard protocol, and fully informed written consents were obtained from all patients. Our study protocol was approved by the Institutional Review Boards of Nagoya University Graduate School of Medicine.

Total RNA isolation and cDNA synthesis. Total RNA was isolated from tissue using TRIzol $^{\mathrm{TM}}$ reagent (Invitrogen, Carlsbad, CA, USA). We added $1000 \mu \mathrm{l}$ of TRIzol reagent to tissue, and the sample was homogenized by Psychotron ${ }^{\mathrm{TM}}$ (Niti-on Co., Ltd., Funabashi, Japan). Next, we incubated the homogenized sample for $5 \mathrm{~min}$ at room temperature prior to the addition of $0.2 \mathrm{ml}$ of chloroform, and they were incubated for 3-min at room temperature. An upper aqueous phase was collected after centrifugation at $12,000 \mathrm{x} \mathrm{g}$ for $15 \mathrm{~min}$ at $4^{\circ} \mathrm{C}$, and mixed with $0.5 \mathrm{ml}$ of $100 \%$ isopropanol. After incubation for $10 \mathrm{~min}$ at room temperature, the sample was centrifuged at $12,000 \mathrm{x} \mathrm{g}$ for $10 \mathrm{~min}$ at $4^{\circ} \mathrm{C}$ and the supernatant was removed. After washing, the pellet with $1 \mathrm{ml}$ of $75 \%$ ethanol, RNA pellets were dried for $30 \mathrm{~min}$, and resuspended in $50 \mu \mathrm{l}$ of RNase-free water and incubated at $55^{\circ} \mathrm{C}$ for $10 \mathrm{~min}$. For serum samples and cell lines, total RNA was extracted using Total RNA Purification ${ }^{\mathrm{TM}}$ kit (Norgen Biotek Corp., Thorold, Ontario, Canada) according to the manufacturer's instructions. We synthesized cDNA from total RNA and extracted genomic DNA using PrimeScript ${ }^{\mathrm{TM}}$ RT reagent kit with gDNA Eraser ${ }^{\mathrm{TM}}$ (Takara Bio, Kusatsu, Japan).

Quantitative real-time RT-PCR. Real-time quantitative PCR (qPCR) was performed with $8 \mathrm{ng}$ of cDNA and SsoAdvanced ${ }^{\mathrm{TM}}$ Universal SYBR-Green Supermix (Bio-Rad Laboratories, Hercules, CA, USA) using CFX Connect ${ }^{\mathrm{TM}}$ Real-Time system (Bio-Rad Laboratories). Specific primers for GALNT13, its variant exon usages, and $G A P D H$ were purchased from SigmaAldrich Japan (Tokyo, Japan). Each primer sequence of wild-type and variant exons is shown in Table I. Cycling conditions were as follows: $30 \mathrm{sec}$ at $95^{\circ} \mathrm{C}$, and then 40 cycles of $5 \mathrm{sec}$ at $95^{\circ} \mathrm{C}$ and $20 \mathrm{sec}$ at $62^{\circ} \mathrm{C}$. Cq values were calculated with Bio-Rad CFX Manager ${ }^{\mathrm{TM}}$ version 2.1. GALNT13 mRNA expression was normalized with an internal control, GAPDH mRNA.

Nested PCR. To detect expression of GALNT13 mRNA in serum sample, nested PCR was performed. Each primer sequence of primary PCR and nested PCR is shown in Table I. First PCR was performed in $50 \mu \mathrm{l}$ using $8 \mathrm{ng}$ cDNA with $1 \mu \mathrm{l}$ of KOD FX ${ }^{\mathrm{TM}}$ (Toyobo Co., Ltd., Osaka, Japan). Nested PCR were done using $1 \mu \mathrm{l}$ of the first PCR product as a template. Both first and nested PCR were done under following conditions: 2 min at $94^{\circ} \mathrm{C}$, and then 40 cycles of $10 \mathrm{sec}$ at $98^{\circ} \mathrm{C}$ and $30 \mathrm{sec}$ at $62^{\circ} \mathrm{C}$. PCR product was visualized on a $1.5 \%$ agarose gel.

Immunohistochemistry. Lung tumor samples were fixed in $4 \%$ paraformaldehyde for $24 \mathrm{~h}$, followed by a solution of $30 \%$ sucrose/PBS until the tissues sank. After embedding in Tissue-Tek ${ }^{\text {TM }}$ O.C.T Compound (Sakura Finetek Japan, Tokyo, Japan), $5 \mu \mathrm{m}$-thick frozen sections were made using Leica CM3050S ${ }^{\mathrm{TM}}$ cryostat (Leica Biosystems, Wetzlar, Germany). The sections treated with $4 \%$ paraformaldehyde for $10 \mathrm{~min}$. Samples were then incubated with $3 \% \mathrm{H}_{2} \mathrm{O}_{2}$ to inhibit endogenous peroxidase activity. In T13 immunostaining, sections were treated with Protein Block Serum-Free ${ }^{\mathrm{TM}}$ (Dako Japan, 

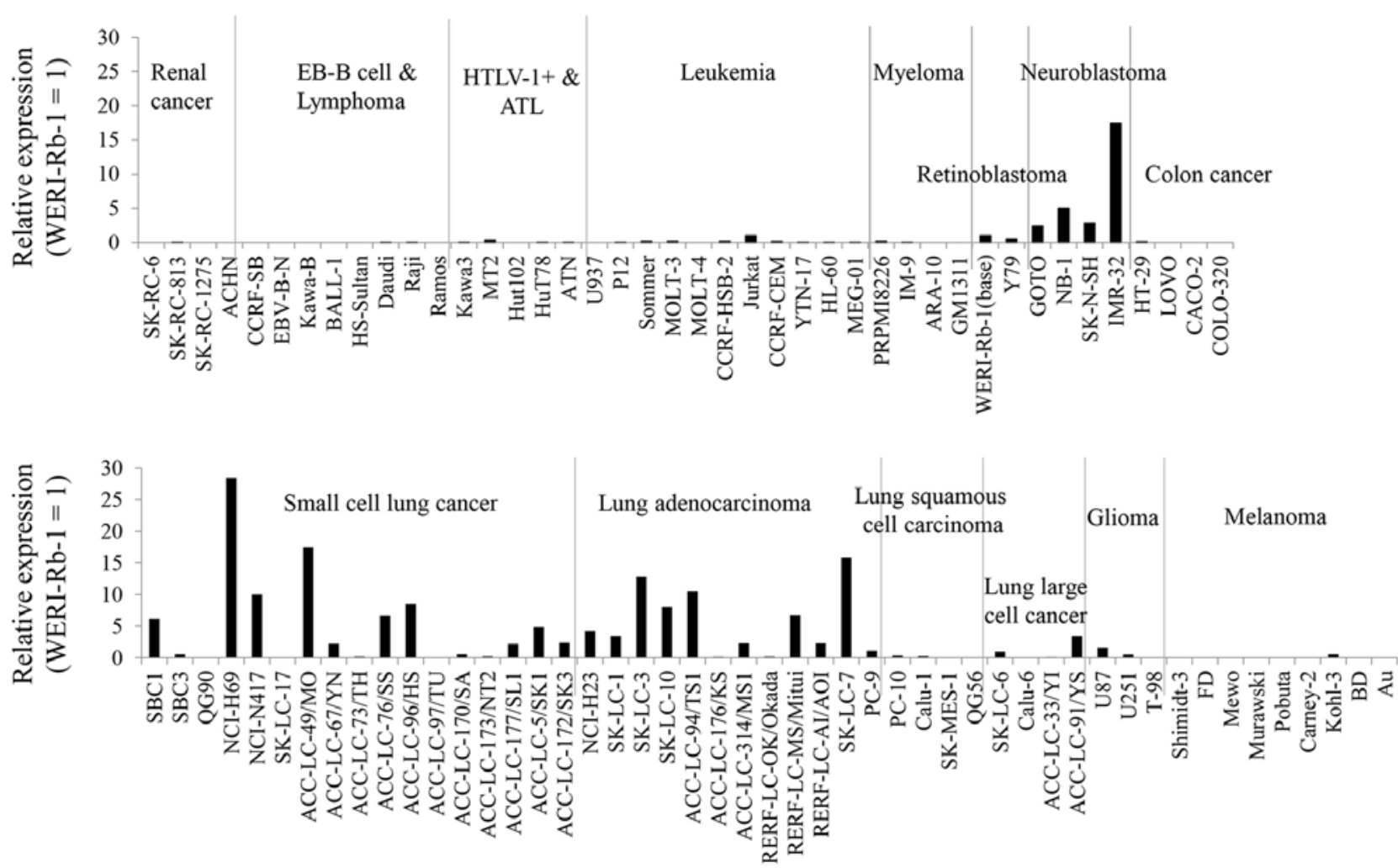

Figure 1. GALNT13 mRNA expression levels in various human cancer cell lines. The expression levels were analyzed by qRT-PCR as described in Materials and methods, and normalized by GAPDH. Relative expression levels obtained by comparing with the value of a control cell line, WERI-Rb-1 were presented. GALNT13 mRNA was found to be highly expressed in lung cancers and neuroblastomas. EB-B, Epstein-Barr virus transformed B cell. HTLV-1+, human T-lymphotropic virus type 1-positive. ATL, adult T-cell leukemia.

Tokyo, Japan) at room temperature for $30 \mathrm{~min}$ in order to block non-specific binding. The sections were incubated at room temperature for $60 \mathrm{~min}$ in $0.4 \mu \mathrm{g} / \mathrm{ml}$ goat polyclonal primary anti-ppGalNAc-T13 antibody (T18) (Santa Cruz Biotechnology, Santa Cruz, CA, USA), followed by applying a secondary biotinylated rabbit anti-goat IgG. In GM1 immunostaining, sections were treated with 3\% BSA in PBS for $15 \mathrm{~min}$ for blocking. Sections were then incubated with cholera toxin B subunit-biotin conjugate (List Biological Laboratories, Campbell, CA, USA) diluted at $0.5 \mu \mathrm{g} / \mathrm{ml}$ with PBS plus $3 \%$ BSA, at room temperature for $60 \mathrm{~min}$. In trimeric Tn antigen immunostaining, after treating with Protein Block Serum-Free ${ }^{\mathrm{TM}}$, the samples were incubated at room temperature for $60 \mathrm{~min}$ with mouse monoclonal primary anti-trimeric Tn antibody (MLS128) $(1.6 \mu \mathrm{g} / \mathrm{ml})$ (provided from Nakada, Kyoto Sangyo University, Kyoto, Japan). Then, a secondary biotinylated horse anti-mouse IgG was applied. The immunoreactivity of these sections was visualized using Vectastain ${ }^{\mathrm{TM}}$ Elite ABC kit (Vector Laboratories, Burlingame, CA, USA) and Dako Liquid $\mathrm{DAB}+$ Substrate Chromogen System ${ }^{\mathrm{TM}}$ (Dako Japan).

Cell culture. The cell line ACC-LC-94/TS1 was derived from human lung adenocarcinoma and cultured in DMEM with 7.5\% fetal bovine serum (FBS; Sigma-Aldrich Japan).

Statistical analysis. The clinicopathological characteristics, overall survival and recurrence-free survival of the patients were obtained from medical records. The relationship was analyzed using the Wilcoxon-Mann-Whitney test. The KaplanMeier method was conducted to estimate survival differences and analyzed using log-rank test. The level of significance was set at $\mathrm{P}<0.05$. These data were analyzed with IBM SPSS Statistics version 22.0 (IBM, Armonk, NY, USA).

\section{Results}

GALNT13 mRNA was highly expressed in human lung cancer cell lines and neuroblastoma cell lines. In order to examine GALNT13 mRNA expression among human tumors, we determined mRNA expression levels in various human cancer cell lines (Fig. 1). From this result, GALNT13 mRNA was found to be highly expressed in lung cancers and neuroblastomas. Among lung cancers, both adenocarcinoma cell lines and small cell lung cancer cell lines showed high expression levels.

Association of GALNT13 expression levels with clinical data of lung cancer patients. Then, to examine whether T13 affects the intensity and prognosis of the disease, we analyzed GALNT13 mRNA expression levels in 91 surgical specimens by real-time RT-PCR, and the results were evaluated by correlating with pathological and clinical data. The GALNT13 mRNA expression levels were normalized by GAPDH mRNA and then relative levels to that of a control cell line (ACCLC-94/TS1, relative expression level=1) were calculated. We divided 91 cases into GALNT13 mRNA high expression group (T13 high, $\mathrm{n}=16$ ) and low expression group ( $\mathrm{T} 13$ low, $\mathrm{n}=75)$ by value 0.001 , cut-off point of sufficient expression levels. 
Table II. Clinicopathological parameters of patients.

\begin{tabular}{|c|c|c|c|}
\hline Characteristic & $\begin{array}{c}\text { T13 high } \\
(\mathrm{N}=16) \\
\text { no }(\%)\end{array}$ & $\begin{array}{l}\mathrm{T} 13 \text { low } \\
(\mathrm{N}=75) \\
\text { no }(\%)\end{array}$ & P-value ${ }^{a}$ \\
\hline Age (years) & & & 0.831 \\
\hline Median & 67 & 67 & \\
\hline Range & $54-78$ & $44-84$ & \\
\hline \multicolumn{4}{|l|}{ Gender } \\
\hline Female & $4(25)$ & $17(23)$ & 0.841 \\
\hline Smoking status & & & 0.343 \\
\hline Never smoked & $3(19)$ & $15(20)$ & \\
\hline Former smoker & $9(56)$ & $52(69)$ & \\
\hline Current smoker & $4(25)$ & $8(11)$ & \\
\hline \multicolumn{4}{|l|}{ Comorbidity } \\
\hline COPD & $5(31)$ & $2(37)$ & 0.648 \\
\hline Diabetes mellitus & $5(31)$ & $12(16)$ & 0.158 \\
\hline Hypertention & $6(38)$ & $29(39)$ & 0.931 \\
\hline \multicolumn{4}{|l|}{ Tumor histologic type } \\
\hline Adenocarcinoma & $10(63)$ & $51(68)$ & 1.000 \\
\hline Non-adenocaricinoma & $6(37)$ & $24(32)$ & \\
\hline UICC-7 stage & & & 0.236 \\
\hline I & $7(44)$ & $45(60)$ & \\
\hline II/III & $9(56)$ & $30(40)$ & \\
\hline pT category & & & 0.124 \\
\hline $0-1$ & $10(63)$ & $31(41)$ & \\
\hline $2-4$ & $6(37)$ & $44(59)$ & \\
\hline pN category & & & 0.062 \\
\hline 0 & $9(56)$ & 59 (79) & \\
\hline $1-2$ & $7(44)$ & $16(21)$ & \\
\hline
\end{tabular}

T13 high, GALNT13 mRNA high expression group; T13 low, GALNT13 mRNA low expression group. Cut-off point was value

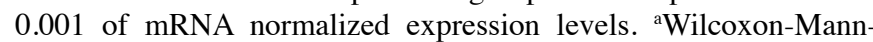
Whitney test.

Clinicopathological parameters of 91 patients are shown in Table II. We found no differences in age, gender, smoking status and comorbidities. Lymph node metastasis tended to be higher in the T13 high group than in T13 low group, but this association was not significant ( $\mathrm{P}=0.062$, Wilcoxon-Mann-Whitney test).

Among the 91 cases, patients with high expression levels of GALNT13 mRNA exhibited reduced overall survival (Fig. 2A). However, the difference in survival rates was not significant $(\mathrm{P}=0.104$; log-rank test). On the other hand, T13 high group was significantly associated with worse prognosis as observed in recurrence-free survival ( $\mathrm{P}=0.045$; Fig. 2B).

It was reported that there were differential usages of exons in GALNT13 RNA sequence, therefore, we chose three variant exons (named E5, E13' E14) and analyzed their expression levels. We obtained base sequence of GALNT13 mRNA and variant exons from GenBank and Ensembl, and designed a specific PCR primer of each. Their exon structures and PCR targets are shown in Fig. 3.
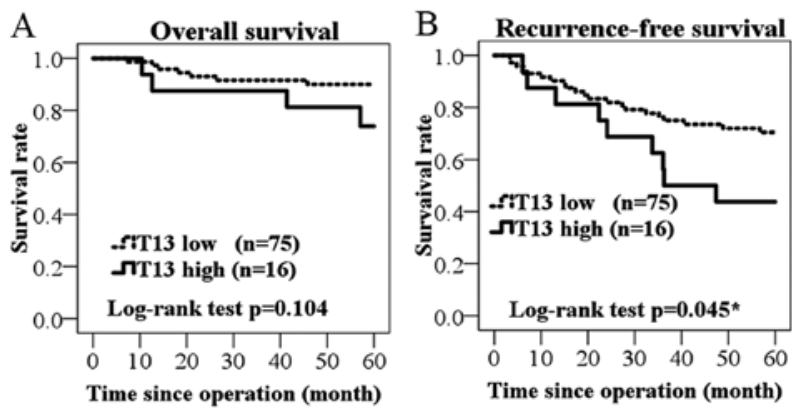

Figure 2. Kaplan-Meier analysis of lung cancer patients for overall survival (A) and recurrence-free survival (B). GALNT13 mRNA high expression group (T13 high) showed a significantly shorter recurrence-free survival compared with the low expression group (T13 low). Patients numbers are shown in the figure. $\mathrm{P}=0.045$. ${ }^{*} \mathrm{P}<0.05$.

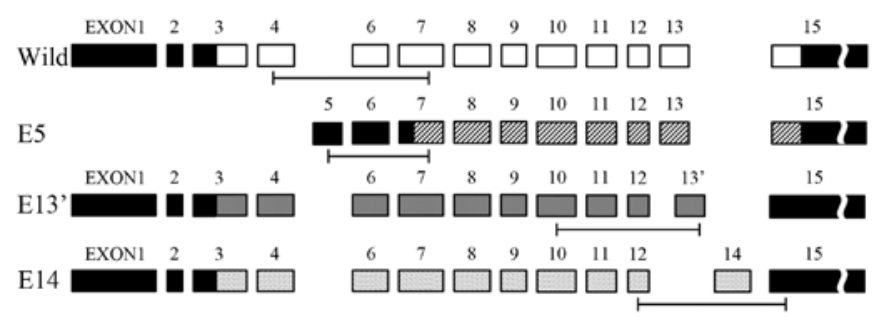

Figure 3. Exon structures of GALNT13 mRNA and its exon usages. These data were obtained from GenBank and Ensembl. We selected three tumor specific variant exon usages named E5, E13' and E14. The black areas are non-cording regions. The lines under individual exon structures indicate PCR-amplified regions by designed primers for detection.

Then, the survival terms of E5, E13' and E14-positive cases were examined by using 40 surgical specimens (Fig. 4). In E5, neither overall survival nor recurrence-free survival was significantly correlated between positive and negative groups $(\mathrm{P}=0.700, \mathrm{P}=0.154$, log-rank test; Fig. 4A). In E13'-positive group, significantly worse prognosis in overall survival, but not in recurrence-free survival was found $(\mathrm{P}=0.042, \mathrm{P}=0.117$, respectively; Fig. 4B). In turn, E14-positive group showed rather better prognosis than negative group, especially in the recurrence-free survival ( $\mathrm{P}=0.019$; Fig. 4C).

Detection of GALNT13 mRNA in sera. We examined whether $\mathrm{T} 13$ in sera can be used as a tumor marker. Some frozen serum samples from surgical patients were analyzed by PCR, but GALNT13 mRNA could not be detected by current analytical condition. Since the expression levels in sera were considered to be less than in tumor tissues, we tried nested PCR, and were able to detect it as shown in Fig. 5A. Sixty-one frozen serum samples from surgical patients and two fresh serum samples from stage IV patients were examined. Among total of 63 serum samples examined by nested PCR, GALNT13 mRNA could be detected in four of the specimens. The parameters of these four patients are shown in Fig. 5B.

Immunohistochemistry analysis revealed that staining of T13 and trimeric Tn antigen significantly correlated with worse prognosis. We evaluated the association between the prognosis and expression of T13, GM1 and trimeric Tn antigen by immunohistochemistry. In a mouse model, we revealed that 
A

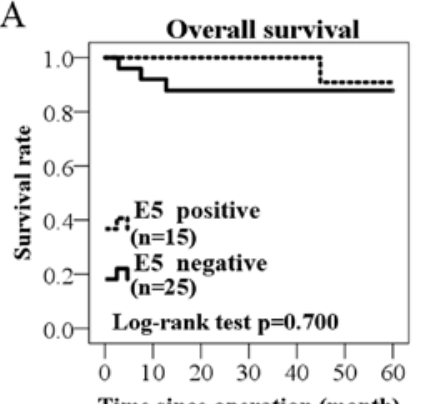

Time since operation (month)

B

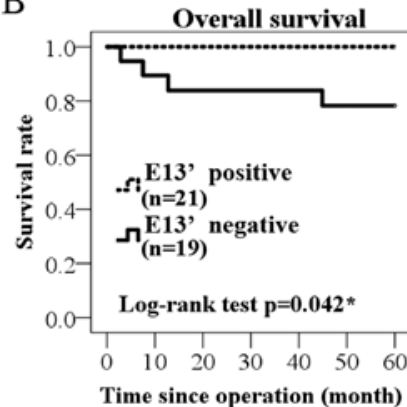

$\mathrm{C}$

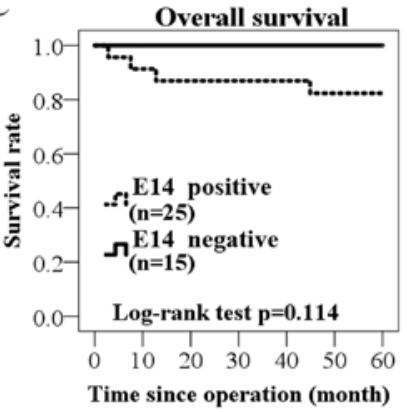

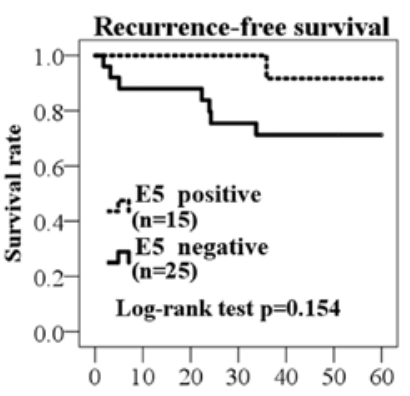

Time since operation (month)

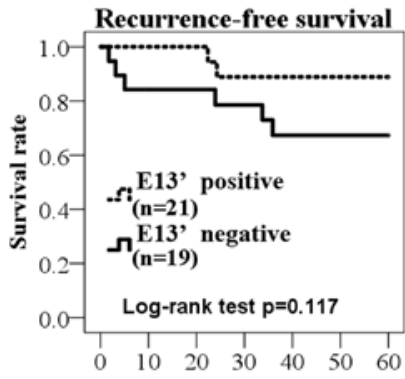

Time since operation (month)

Recurrence-free survival

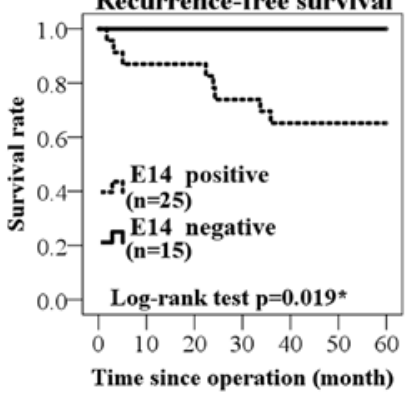

Figure 4. Kaplan-Meier analysis for overall and recurrence-free survival of patients having GALNT13 RNA variant exon usages: E5 (A), E13' (B) and E14 (C). E13'-positive expression group was significantly associated with worse prognosis in overall survival. Conversely, E14-positive expression group showed significantly prolonged recurrence-free survival compared with the negative group. Number of patients in each group is shown in the figure.

reduced expression level of GM1 resulted in the upregulation of GALNT13 gene, and T13 formed trimeric Tn antigen on Syndecan-1, leading to increased adhesion to extracellular matrix (20). Therefore, we also examined the correlation between T13, GM1 and trimeric Tn in lung cancer tissues. The tumor samples which showed positive staining in more than $10 \%$ of tumor cells were defined as positive expression. Images of the positive and negative staining of T13, GM1 and trimeric Tn are shown in Fig. 6. We analyzed 35 samples (patients received operation in May, 2008-December, 2008) and evaluated the correlation between the staining and the patients prognosis. We found that the T13 positive group tended to show worse overall survival than T13-negative group without significance $(\mathrm{P}=0.248$, log-rank test; Fig. 7A). On the other hand, T13-positive group had strongly significant association with shortened recurrence-free survival $(\mathrm{P}=0.007)$. This result was similar to results of GALNT13 mRNA expression analysis. As for GM1, GM1 positive group tended to show longer survival in overall and recurrence-free survival, but it was not significant $(\mathrm{P}=0.180, \mathrm{P}=0.061$; Fig. $7 \mathrm{~B})$. For trimeric Tn antigen, the positive group showed a trend of short survival,

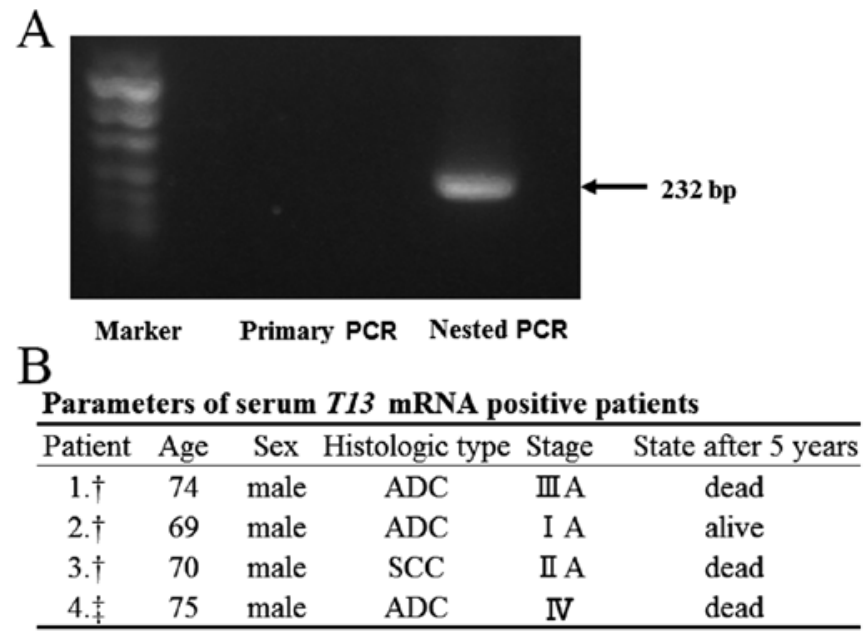

Figure 5. Detection of GALNT13 mRNA from patient serum samples by nested PCR. (A) Agarose gel electrophoresis of PCR products. Design of primers and qPCR are described in Table I. The 310 bp primary PCR product was negative in the central lane, but the $232 \mathrm{bp}$ nested PCR product was positive on the right. (B) Sixty-one frozen serum samples from surgical patients and two fresh serum samples from stage 4 patients were evaluated by nested PCR. GALNT13 mRNA could be detected in four of the specimen. The age, gender, histologic type, stage and state after five years of the four patients are shown. T13, GALNT13. 'Surgical patient. ${ }^{\star}$ Stage 4 patient.

Table III. Correlation between T13, GM1 and trimeric Tn.

\begin{tabular}{lccc}
\hline & $\begin{array}{c}\text { T13 positive } \\
(\mathrm{N}=13)\end{array}$ & $\begin{array}{c}\text { T13 negative } \\
(\mathrm{N}=22)\end{array}$ & P-value $^{\mathrm{a}}$ \\
\hline GM1 & & & $0.007^{\mathrm{b}}$ \\
Positive & 0 & 9 & \\
Negative & 13 & 13 & $0.006^{\mathrm{b}}$ \\
Trimeric Tn & & 6 & \\
Positive & 10 & 16 & \\
Negative & 3 & &
\end{tabular}

T13, ppGalNAc-T13. ${ }^{\text {F}}$ Fisher's exact test; ${ }^{\mathrm{b}} \mathrm{P}<0.01$.

and there was a significant difference in the recurrence-free survival ( $\mathrm{P}=0.007$; Fig. $7 \mathrm{C}$ ). The correlation between T13, GM1, and trimeric Tn expression is shown in Table III. There was a negative correlation between T13 and GM1 expression ( $\mathrm{P}=0.007$, Fisher's exact test). By contrast, positive correlation was observed in T13 and trimeric Tn expression $(\mathrm{P}=0.006)$.

\section{Discussion}

In the present study, we demonstrated that T13 expression was significantly associated with the worse prognosis of lung cancer patients. In pathological data, lymph node metastasis, which was a prognostic factor in resected non-small cell lung cancer (22), tended to be higher in the GALNT13 mRNA high expression group. From these results, it can be considered that T13 plays an important role in the malignancy of cancer, particularly in metastasis process in lung cancers. This speculation is supported by our previous study, which showed that 

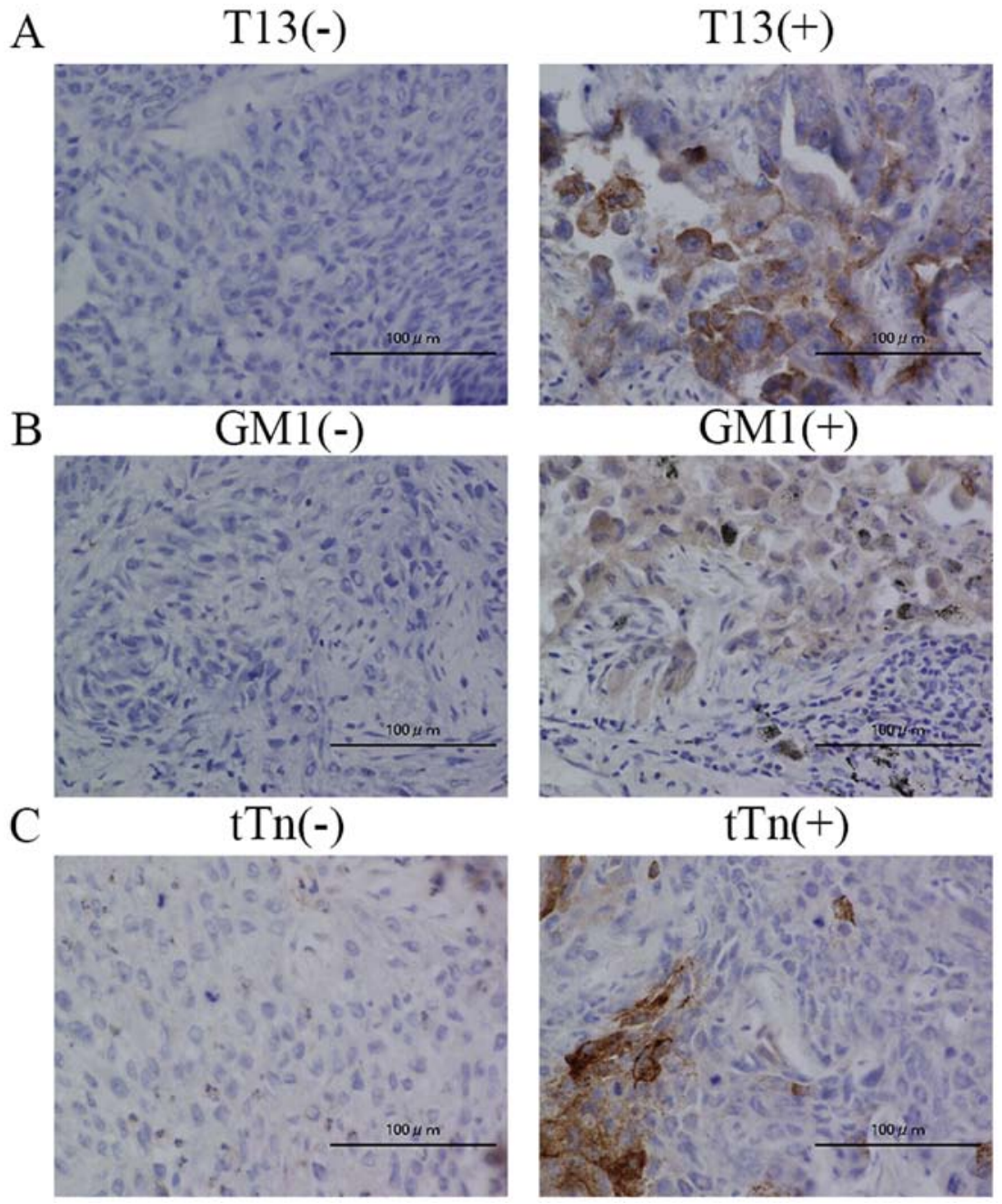

Figure 6. Expression of T13, GM1 and trimeric Tn antigen in lung cancer tissues as analyzed by immunohistochemistry. (A-C) Left panel is negative expression tissue and right panel is positive expression tissue of each staining by anti-ppGalNAc-T13 (T18) (A), cholera toxin B subunit (B), and anti-trimeric Tn antibody (MLS128) (C), respectively. Scale bars, $100 \mu \mathrm{m}$. T13, ppGalNAc-T13; tTn, trimeric Tn.

T13 induces high metastatic potential of murine Lewis lung cancer. In the study, we also revealed that T13 was upregulated as a result of reduced GM1, leading to enhanced metastasis by formation of trimeric Tn antigen on Syndecan 1 in mouse Lewis lung cancer $(19,20)$. In this study, we found the negative correlation of GM1 and T13, and the positive correlation of trimeric Tn and T13 by immunohistochemistry. These results corresponded with our previous data in the experimental mouse metastasis model.

In a previous study, expression levels of GALNT13 mRNA were analyzed in various adult and fetal human tissues. The expression level was highest in the fetal brain, followed by the adult brain. GALNT13 mRNA was expressed at minimal or undetectable levels in the other tissues (18). It was also shown that T13 was able to form trimeric Tn antigen, and significant decrease in Tn antigen expression was found in the cerebellum of the T13 knockout mouse (18). However, roles of T13 have not been clarified. In the analysis of GALNT13 mRNA expression levels among human tumor cell lines, we found that the expression levels were higher in only lung cancer and neuroblastoma lines (Fig. 1). Combined with the fact that GALNT13 mRNA was scarcely expressed in normal lung tissues, T13 may be involved in the mechanism for evolution or malignant prop- erties of lung cancers. We also demonstrated the association between T13 and metastatic potential and/or poor prognosis of lung cancer patients. Considering that $\mathrm{T} 13$ is mainly expressed in fetal brain, T13 and its product trimeric Tn may be involved in the cell growth or proliferation. In fact, it was reported that MLS128 monoclonal antibody, which binds an epitope consisting of three consecutive Tn-antigens, inhibited colon and breast cancer cell growth (23-25).

We also analyzed several variant exon usages in GALNT13 mRNA sequence, and found that one variant exon expression had significant association with worse prognosis (Fig. 4). By contrast, another variant exon-positive group showed better prognosis than negative group. This was an interesting and surprising result. GALNT13 mRNA differential usages of exons have different sequences of lectin like domain. Therefore, the reason for opposite result among variant exon usages may be attributed to the difference in the recognition of the substrate during the synthesis of O-glycans (26). Thus, tumor-specific and malignant property-associated variant exon usage may be important as targets for molecular therapy of cancers, although precise mechanisms remain to be investigated.

We demonstrated that T13 and trimeric Tn antigen had a relationship with worse prognosis of lung cancer patients by 

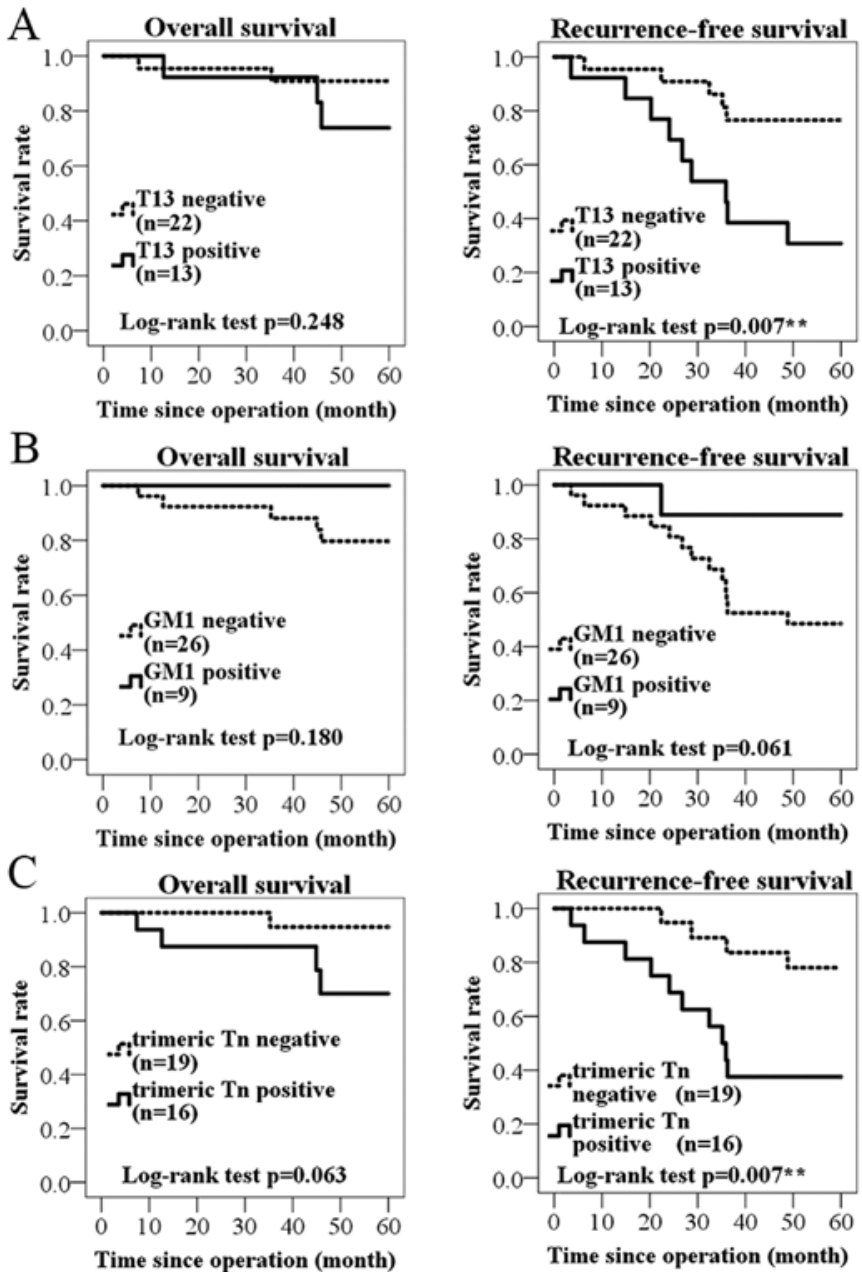

Figure 7. Kaplan-Meier analysis for overall and recurrence-free surviva between positive and negative groups for T13 (A), GM1 (B), and trimeric Tn (C) in immunohistochemistry. (A) T13-positive group showed significantly worse prognosis in overall survival. (B) GM1-positive group showed longer survival, but it was not significant. (C) Trimeric Tn antigen-positive group showed significantly shorter survival in recurrence-free survival. T13, ppGalNAc-T13; tTn, trimeric Tn. ${ }^{* *} \mathrm{P}<0.01$.

immunohistochemistry. This result also suggests that T13 and trimeric Tn antigen can be used as a tumor marker. Although GALNT13 mRNA could be detected in serum sample, the expression levels were too low to be stably quantified. If the carrier proteins of T13 or trimeric Tn are identified, we can more easily detect them in serum by using ELISA. Thus, they can be expected as a tumor marker, leading to early detection of lung cancers or minimal metastatic diseases.

The limitation of the present study is partly due to restricted selection of specimens. GALNT13 mRNA detection and immunohistochemistry were conducted in primary tumors obtained in surgery. Because we did not get metastatic tumors and lymph nodes, we could not examine the difference in the expression levels between primary and metastatic tumors. Despite these study limitations, we demonstrated that higher expression of T13 in primary tumors were associated with the poor prognosis. Consequently, it can be an indicator for whether we should perform postoperative therapy and careful observation. That is because postoperative therapy largely affect patient survival, while a part of patients can obtain benefit $(27,28)$. Therefore, by using specific antibodies, T13 and trimeric Tn antigen might be expected as a new target of molecular treatment.

In conclusion, the present study showed that high expression levels of GALNT13 mRNA is associated with poor prognosis of lung cancer patients by using quantitative real-time RT-PCR. Furthermore, T13 and trimeric Tn antigen expression were strongly correlated with shortened survival in immunostaining. Our results suggest that T13 might be a useful prognostic factor and might be a new target for cancer treatment.

\section{Acknowledgements}

We thank T. Mizuno, Y. Nakayasu and N. Hattori for technical assistance and K. Ushida for technical teaching of histochemical analyses. The present study was supported by Grants-in-Aid of the Ministry of Education, Culture, Sports, Science and Technology of Japan (MEXT) (15H04696, 15K15080, 25670141 and 24390078).

\section{References}

1. Siegel RL, Miller KD and Jemal A: Cancer statistics, 2015. CA Cancer J Clin 65: 5-29, 2015.

2. Rosell R, Moran T, Queralt C, Porta R, Cardenal F, Camps C, Majem M, Lopez-Vivanco G, Isla D, Provencio M, et al; Spanish Lung Cancer Group: Screening for epidermal growth factor receptor mutations in lung cancer. N Engl J Med 361: 958-967, 2009.

3. Solomon B, Varella-Garcia M and Camidge DR: ALK gene rearrangements: A new therapeutic target in a molecularly defined subset of non-small cell lung cancer. J Thorac Oncol 4: 1450-1454, 2009.

4. Hakomori S: Cancer-associated glycosphingolipid antigens: Their structure, organization, and function. Acta Anat (Basel) 161: 79-90, 1998.

5. Kasahara K and Sanai Y: Possible roles of glycosphingolipids in lipid rafts. Biophys Chem 82: 121-127, 1999.

6. Fuster MM and Esko JD: The sweet and sour of cancer: Glycans as novel therapeutic targets. Nat Rev Cancer 5: 526-542, 2005.

7. Li M, Song L and Qin X: Glycan changes: Cancer metastasis and anti-cancer vaccines. J Biosci 35: 665-673, 2010.

8. Wiegandt H: Gangliosides. In: Glycolipids. Vol. 10. Wiegandt $\mathrm{H}$ (ed). Elsevier Science Publishers, Amsterdam, pp199-260, 1985.

9. Cheresh DA, Rosenberg J, Mujoo K, Hirschowitz L and Reisfeld RA: Biosynthesis and expression of the disialoganglioside GD2, a relevant target antigen on small cell lung carcinoma for monoclonal antibody-mediated cytolysis. Cancer Res 46: 5112-5118, 1986.

10. Yoshida S, Fukumoto S, Kawaguchi H, Sato S, Ueda R and Furukawa K: Ganglioside $G_{D 2}$ in small cell lung cancer cell lines: Enhancement of cell proliferation and mediation of apoptosis. Cancer Res 61: 4244-4252, 2001

11. Yoshida S, Kawaguchi H, Sato S, Ueda R and Furukawa K: An anti-GD2 monoclonal antibody enhances apoptotic effects of anti-cancer drugs against small cell lung cancer cells via JNK (c-Jun terminal kinase) activation. Jpn J Cancer Res 93: 816-824, 2002.

12. Alfonso S, Valdés-Zayas A, Santiesteban ER, Flores YI, Areces F, Hernández M, Viada CE, Mendoza IC, Guerra PP, García E, et al: A randomized, multicenter, placebo-controlled clinical trial of racotumomab-alum vaccine as switch maintenance therapy in advanced non-small cell lung cancer patients. Clin Cancer Res 20: 3660-3671, 2014

13. Kannagi R: Molecular mechanism for cancer-associated induction of sialyl Lewis X and sialyl Lewis A expression-The Warburg effect revisited. Glycoconj J 20: 353-364, 2004.

14. Brockhausen I: Mucin-type O-glycans in human colon and breast cancer: Glycodynamics and functions. EMBO Rep 7: 599-604, 2006.

15. Cao Y, Stosiek P, Springer GF and Karsten U: ThomsenFriedenreich-related carbohydrate antigens in normal adult human tissues: A systematic and comparative study. Histochem Cell Biol 106: 197-207, 1996. 
16. Ju T, Otto VI and Cummings RD: The Tn antigen-structural simplicity and biological complexity. Angew Chem Int Ed Engl 50: 1770-1791, 2011.

17. Zhang Q, Furukawa K, Chen HH, Sakakibara T, Urano T and Furukawa K: Metastatic potential of mouse Lewis lung cancer cells is regulated via ganglioside GM1 by modulating the matrix metalloprotease-9 localization in lipid rafts. J Biol Chem 281: $18145-18155,2006$.

18. Zhang Y, Iwasaki H, Wang H, Kudo T, Kalka TB, Hennet T, Kubota T, Cheng L, Inaba N, Gotoh M, et al: Cloning and characterization of a new human UDP-N-acetyl- $\alpha-D$ galactosamine:polypeptide $\mathrm{N}$-acetylgalactosaminyltransferase, designated pp-GalNAc-T13, that is specifically expressed in neurons and synthesizes GalNAc $\alpha$-serine/threonine antigen. J Biol Chem 278: 573-584, 2003.

19. Matsumoto Y, Zhang Q, Akita K, Nakada H, Hamamura K, Tokuda N, Tsuchida A, Matsubara T, Hori T, Okajima T, et al: pp-GalNAc-T13 induces high metastatic potential of murine Lewis lung cancer by generating trimeric Tn antigen. Biochem Biophys Res Commun 419: 7-13, 2012.

20. Matsumoto Y, Zhang Q, Akita K, Nakada H, Hamamura K, Tsuchida A, Okajima T, Furukawa K, Urano T and Furukawa K: Trimeric Tn antigen on syndecan 1 produced by ppGalNAcT13 enhances cancer metastasis via a complex formation with integrin $\alpha 5 \beta 1$ and matrix metalloproteinase 9. J Biol Chem 288 . 24264-24276, 2013.

21. Berois N, Blanc E, Ripoche H, Mergui X, Trajtenberg F, Cantais S, Barrois M, Dessen P, Kågedal B, Bénard J, et al: ppGalNAc-T13: A new molecular marker of bone marrow involvement in neuroblastoma. Clin Chem 52: 1701-1712, 2006.

22. Naruke T, Suemasu K and Ishikawa S: Lymph node mapping and curability at various levels of metastasis in resected lung cancer. J Thorac Cardiovasc Surg 76: 832-839, 1978.

23. Morita N, Yajima Y, Asanuma H, Nakada H and FujitaYamaguchi Y: Inhibition of cancer cell growth by anti-Tn monoclonal antibody MLS128. Biosci Trends 3: 32-37, 2009.
24. Zamri N, Masuda N, Oura F, Yajima Y, Nakada H and FujitaYamaguchi Y: Effects of two monoclonal antibodies, MLS128 against $\mathrm{Tn}$-antigen and $1 \mathrm{H} 7$ against insulin-like growth factor-I receptor, on the growth of colon cancer cells. Biosci Trends 6 : 303-312, 2012.

25. Zamri N, Masuda N, Oura F, Kabayama K, Yajima Y, Nakada H, Yamamoto K and Fujita-Yamaguchi Y: Characterization of anti-Tn-antigen MLS128 binding proteins involved in inhibiting the growth of human colorectal cancer cells. Biosci Trends 7: 221-229, 2013.

26. Gerken TA, Revoredo L, Thome JJ, Tabak LA, VesterChristensen MB, Clausen H, Gahlay GK, Jarvis DL, Johnson RW, Moniz HA, et al: The lectin domain of the polypeptide GalNAc transferase family of glycosyltransferases (ppGalNAc Ts) acts as a switch directing glycopeptide substrate glycosylation in an $\mathrm{N}$ - or C-terminal direction, further controlling mucin type O-glycosylation. J Biol Chem 288: 19900-19914, 2013.

27. Keller SM, Adak S, Wagner H, Herskovic A, Komaki R, Brooks BJ, Perry MC, Livingston RB and Johnson DH; Eastern Cooperative Oncology Group: A randomized trial of postoperative adjuvant therapy in patients with completely resected stage II or IIIA non-small-cell lung cancer. N Engl J Med 343: 1217-1222, 2000

28. Winton T, Livingston R, Johnson D, Rigas J, Johnston M, Butts C, Cormier Y, Goss G, Inculet R, Vallieres E, et al; National Cancer Institute of Canada Clinical Trials Group; National Cancer Institute of the United States Intergroup JBR.10 Trial Investigators: Vinorelbine plus cisplatin vs. observation in resected non-small-cell lung cancer. N Engl J Med 352: 2589-2597, 2005. 